\title{
Unfolding Thermodynamics of the Tetrameric Chaperone, $\mathrm{Sec}^{\dagger}$
}

\author{
Vikram G. Panse, $\$$ Chittor P. Swaminathan, \\ Molecular Biophysics Unit, Indian Institute of Science, Bangalore 560 012, India, and Chemical Biology Unit, \\ Jawaharlal Nehru Center for Advanced Scientific Research, Jakkur P.O., Bangalore 560 064, India
}

Received October 26, 1999; Revised Manuscript Received December 6, 1999

\begin{abstract}
SecB is a cytosolic tetrameric chaperone in Escherichia coli, which maintains polypeptides, destined for export in a translocation competent state. The thermodynamics of unfolding of SecB was studied as a function of protein concentration, by using high sensitivity-differential scanning calorimetry and spectroscopic methods. The thermal unfolding of tetrameric SecB is reversible and can be well described as a two-state transition in which the folded tetramer is converted directly to unfolded monomers. Increasing the $\mathrm{pH}$ decreases the stability of the tetramer significantly, the $T_{\mathrm{m}}$ changing from $341.3 \mathrm{~K}$ at $\mathrm{pH} 6.5$ to $332.6 \mathrm{~K}$ at pH 9.5. The value of $\Delta C_{\mathrm{p}}$ obtained from measurements of $\Delta H_{\mathrm{m}}$ as a function of $T_{\mathrm{m}}$ was 10.7 $\pm 0.7 \mathrm{kcal} \mathrm{mol}^{-1} \mathrm{~K}^{-1}$. The value of $\Delta C_{\mathrm{p}}$ is among the highest measured for a multimeric protein. At 298 $\mathrm{K}, \mathrm{pH} 7.4$, the $\Delta G^{\circ}$ for the SecB tetramer is $27.9 \pm 2 \mathrm{kcal} \mathrm{mol}^{-1}$. Denaturant-mediated unfolding of $\mathrm{SecB}$ was found to be irreversible. The reactivity of the four solvent-exposed free thiols in tetrameric $\mathrm{SecB}$ is salt dependent. The kinetics of reactivity suggests that these four cysteines are in close proximity to each other and that these residues on each monomer are in chemically identical environments. The thermodynamic data suggest that $\mathrm{SecB}$ is a stable, well-folded, and tightly packed tetramer and that substrate binding occurs at a surface site rather than at an interior cavity.
\end{abstract}

The folding of newly synthesized proteins inside the cell depends on a set of conserved proteins known as molecular chaperones $(1,2)$. Molecular chaperones do not contain information specifying correct folding, rather they prevent nonproductive interactions between nonnative polypeptide chains and typically increase the yield but not the rate of folding reactions (2). In Escherichia coli, export of proteins from the cytoplasm into the periplasm and the outer membrane involves transport across the inner membrane. The export of a subset of periplasmic proteins is aided by the cytoplasmic chaperone SecB (3). Formation of stable folded structure inhibits translocation of proteins across the inner membrane $(4,5)$. SecB is a homotetrameric chaperone having a molecular mass of $68 \mathrm{kDa}$ and is known to form stoichiometric complexes with precursor proteins (6). SecB is thought to bind to unfolded and partially folded polypeptides in the cytosol and mediate their entry into the export pathway (3). SecB has been shown to bind to a variety of proteins in the unfolded, molten globule and partially folded states but not to the native state $(7-9)$.

Knowledge of the origins of the protein stability is essential to the understanding of structure and function. One of the methods to quantify stability of a protein is to populate the native and unfolded states by physical and chemical means and analyze the transitions as measured by calorimetric or spectroscopic methods (10). A large body of work on the

Financial support from Department of Biotechnology, Government of India, DBT Grant BT/R and D/15/09/1993.

* To whom correspondence should be addressed. E-mail: varadar@mbu.iisc.ernet.in. Fax: 91-80-3600683 or 3600535. Phone: 91-80-3092612.

$\doteqdot$ Indian Institute of Science.

$\S$ Jawaharlal Nehru Center for Advanced Scientific Research. thermodynamics of small monomeric proteins has suggested that the hydrophobic effect and loss of conformational entropy are the major determinants of stability of the native state $(11,12)$. In contrast, there are fewer studies on multimeric proteins, and in the majority of the cases, they do not exhibit reversible denaturation. In addition to interactions within the polypeptide chain, oligomeric proteins are characterized by intersubunit interactions, which may contribute to the overall stability. In addition to providing the energetics of the unfolding process, equilibrium-unfolding studies can provide insights into the events that occur during the assembly of oligomers from individual chains. Thermal unfolding studies of multimeric proteins such as GroES, p53, and HIV protease have emphasized the role of quaternary association in the stabilization of the multimeric assembly $(13-16)$.

In this work, we have examined the thermal and denaturant-mediated unfolding of SecB by means of $\mathrm{DSC}^{1}$ and spectroscopic methods to gain insights into the thermodynamics and mechanism of disassembly of the tetrameric structure. We show that the thermal unfolding of the SecB tetramer is reversible and involves a single highly cooperative transition from the native tetrameric state to unfolded monomers, demonstrating that the unfolding of the protein is linked to subunit dissociation. We have also examined

${ }^{1}$ Abbreviations: CD, circular dichroism; DSC, differential scanning calorimetry; DTNB, 4-dithionitrobenzoic acid; DTP, 4-dithiopyridine; IPTG, 1-isopropyl 1-thio- $\beta$-D-galactopyranoside; PMSF, phenylmethanesulfonyl fluoride; TP, 4-thiopyridine; TNB, 4-thionitrobenzene; CGH10, citrate-glycine-HEPES buffer (10 $\mathrm{mM}$ each); $\Delta C_{\mathrm{p}}$, change in heat capacity; $\mathrm{GdnHCl}$, guanidine hydrochloride; $T_{\mathrm{m}}$, temperature at which fraction of unfolded protein is $0.5 ; \Delta H_{\mathrm{m}}$, molar unfolding enthalpy at $T_{\mathrm{m}}, \Delta H_{\mathrm{v}}$, van't Hoff enthalpy; $\Delta G^{\circ}{ }_{\mathrm{u}}$, Gibbs' free-energy change upon unfolding. 
the reaction kinetics of the four solvent exposed free thiols in the SecB tetramer with 4-dithionitrobenzoic acid and 4-dithiopyridine. The kinetic data suggests that the cysteines are in close proximity and the correlation of this reactivity to the symmetry of the tetramer is discussed.

\section{EXPERIMENTAL PROCEDURES}

Materials. PMSF, iodoacetamide, IPTG, DTT, DTNB, and DTP were from Sigma (Sigma Chemical Company, Missouri). Fast flow Q-Sepharose and Sephacryl S-200 were from Pharmacia. Ultrapure grades of $\mathrm{GdnHCl}$ and Tris were purchased from GIBCOBRL. All other chemicals were of analytical grade.

Protein Purification. The SecB expression plasmid pJW25 in strain BL21 (DE3) was obtained from Prof. B. de Kruijff. SecB purification was done as reported previously (9). The purified protein was estimated to be $99 \%$ pure by SDSPAGE as detected by silver staining (17) and analytical gel filtration HPLC. The extinction coefficient at $280 \mathrm{~nm}$ was taken to be $11900 \mathrm{M}^{-1} \mathrm{~cm}^{-1}$ for monomeric SecB (18). All the $\mathrm{SecB}$ concentrations reported here are tetrameric unless otherwise stated.

Spectroscopic Measurements. Thermal unfolding of SecB monitored by optical probes was carried out using a JASCO J-500 spectropolarimeter, JASCO UV/VIS 7850 spectrophotometer, and in a JASCO FP-777 spectrofluorimeter. The changes in secondary structure were followed by wavelength scans from 200 to $250 \mathrm{~nm}$ at various concentrations of protein and were performed in a $5 \mathrm{~mm}$ rectangular quartz cuvette at $1{ }^{\circ} \mathrm{C} \mathrm{min}-1$. Changes in tertiary structure were monitored by scanning from 300 to $250 \mathrm{~nm}$ in a $1 \mathrm{~cm}$ path length quartz cuvette at $1^{\circ} \mathrm{C} \mathrm{min}^{-1}$. Changes in absorbance upon thermal unfolding were monitored using a $1 \mathrm{~cm}$ path length cuvette placed in a thermo-coupled device with a stirring assembly for heating the sample at $1{ }^{\circ} \mathrm{C} \mathrm{min}-1$. Unfolding transitions obtained by spectroscopic methods were analyzed as described (14) to obtain $F_{\mathrm{u}}$. Reversibility of SecB unfolding as monitored by fluorescence emission was carried out using $1 \mu \mathrm{M}$ of native and refolded SecB. Denaturant-mediated unfolding was carried out using urea and guanidine hydrochloride in CGH10 buffer, $\mathrm{pH}$ 7.4. SecB $(1 \mu \mathrm{M})$ was incubated in CGH10 overnight in varying concentrations of denaturants and the equilibrium unfolding monitored by measuring the change in fluorescence intensity at $353 \mathrm{~nm}$ with excitation used at $280 \mathrm{~nm}$.

Differential Scanning Calorimetry. All calorimetric experiments were carried out in a Microcal MC-2 ultrasensitive calorimeter. DSC measurements were carried out as a function of scan rate, protein concentration, and $\mathrm{pH}$. The data were analyzed using Origin software supplied by the DSC manufacturer. The raw data of the calorimetric scans were baseline corrected and concentration normalized. Progress baseline connection of the pre- and posttransition baselines was generated for estimation of the heat capacity change. Analysis of the DSC scans was done using the single two-state transition with subunit dissociation model, as described in the manual supplied by the manufacturer. All protein solutions were dialyzed extensively against large volumes of desired buffer and degassed prior to use. The sample $\mathrm{pH}$ was checked before and after the scan. The buffer used was CGH10 for the entire $\mathrm{pH}$ range 6.5-9.5.
DTNB and DTP Assays. DTNB assays were carried out in a JASCO spectrophotometer. For monitoring the unfolding kinetics of SecB, $100 \mu \mathrm{L}$ solution of $6.6 \mu \mathrm{M}$ solution of SecB was added to a $500 \mu \mathrm{L}$ solution of $200 \mu \mathrm{M}$ DTNB in denaturant contained in CGH10, $\mathrm{pH}$ 7.4. The reactivity of Cys residues to DTNB was monitored by measuring the increase in absorbance at $412 \mathrm{~nm}$. The molar extinction coefficient of TNB released in the reaction was taken to be $13600 \mathrm{M}^{-1} \mathrm{~cm}^{-1}$ (19). For equilibrium experiments, 700 $\mu \mathrm{L}$ of a $1.1 \mu \mathrm{M}$ solution of SecB was incubated overnight either in $\mathrm{GdnHCl}$ or urea contained in CGH10, $\mathrm{pH}$ 7.4. The number of free thiols labeled were measured $4 \mathrm{~h}$ after adding $20 \mu \mathrm{L}$ of a $5 \mathrm{mM}$ solution of DTNB to SecB. For salt dependence of the reactivity of the solvent-exposed Cys, the concentration of $\mathrm{KCl}$ in $\mathrm{CGH} 10, \mathrm{pH} 7.4$, was varied from 50 to $900 \mathrm{mM}$. A total of $20 \mu \mathrm{L}$ of a $5 \mathrm{mM}$ solution of DTNB in CGH10, pH 7.4, was added, and the increase in absorbance at $412 \mathrm{~nm}$ was monitored as a function of time. DTP assays were carried out in an identical manner to that stated above except that $0.7 \mu \mathrm{M}$ of SecB was used and the release of the thio-pyridine moiety was followed at $324 \mathrm{~nm}$. The molar extinction coefficient of the released TP moiety was taken to be $19600 \mathrm{M}^{-1} \mathrm{~cm}^{-1}$ (19).

Gel Filtration Experiments. Gel filtration experiments were carried out in a Superose 6 column (Pharmacia) with a flow rate of $0.5 \mathrm{~mL} \mathrm{~min}{ }^{-1}$. The buffer used was CGH10 with $200 \mathrm{~mm} \mathrm{NaCl}$. The column was equilibrated with the denaturant prepared in CGH10 and $200 \mathrm{mM} \mathrm{NaCl}$. The refractive index of the eluate solution was measured to check the equilibration of the column with the denaturant (20). A total of $100 \mu \mathrm{g}$ of protein in $20 \mu \mathrm{L}$ of denaturant in CGH10 $\mathrm{pH} 7.4$ incubated overnight was injected, and the elution of the protein was monitored by absorbance at $280 \mathrm{~nm}$.

\section{RESULTS AND DISCUSSION}

Thermodynamic Parameters Characterizing SecB Stability. We have characterized the stability of SecB as function of protein concentration and $\mathrm{pH}$ by high-sensitivity DSC, fluorescence, and absorption spectroscopy. The transitions were reversible under all conditions studied, as demonstrated by repeated scans of the same sample. Figure 1 shows the buffer-corrected partial molar excess heat capacity data for $86.4 \mu \mathrm{M}$ of SecB in CGH10, $\mathrm{pH} 7.4$, scanned at $60^{\circ} \mathrm{C} \mathrm{h}^{-1}$. The dotted line indicates the calculated baseline excess heat capacity of the system. Examination of the areas under the scan and rescan reveals a high reversibility of $83 \%$. The effect of scan rate was studied by carrying out DSC scans at $20{ }^{\circ} \mathrm{C} \mathrm{h}^{-1}$. The data indicate that both $T_{\mathrm{m}}$ and $\Delta H_{\mathrm{m}}$ are independent of scan rate. For all DSC scans and optical measurements, the transition temperature is defined as the temperature at which $F_{\mathrm{u}}=0.5$. Figure 2 shows a set of DSC scans obtained in the protein concentration range 16.9-86.4 $\mu \mathrm{M}$ in CGH10, $\mathrm{pH}$ 7.4. The thermodynamic parameters characterizing unfolding, at different concentrations of SecB, are summarized in Table 1. It is clear from Figure 3 that the transition temperature increases with protein concentration. At the highest concentration tested of $86.4 \mu \mathrm{M}$, the transition occurs at $344.1 \mathrm{~K}$ with an excess heat capacity change of $12.9 \mathrm{kcal} \mathrm{mol}^{-1} \mathrm{~K}^{-1}$. Each of the DSC scans was analyzed as described above. The value of $n$ (number of subunits) was systematically changed so as to get the value of $n$ that best fits the experimental data. A value of $n=4$ best described 


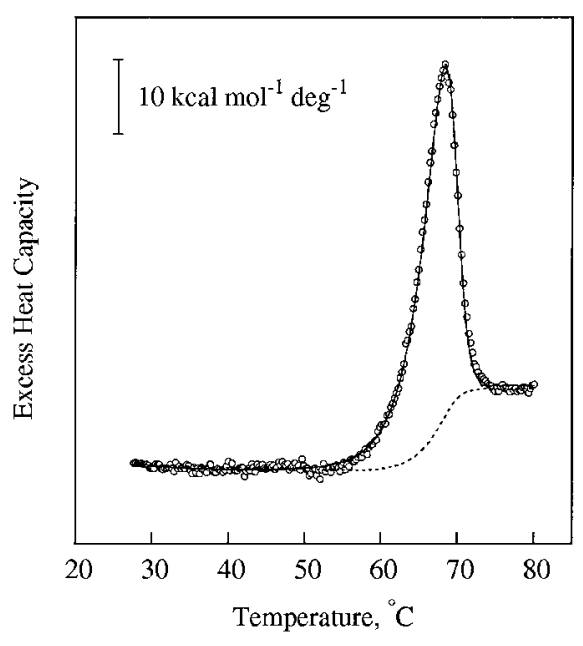

Figure 1: Representative DSC scan of $86.4 \mu \mathrm{M}$ of SecB at $\mathrm{pH}$ 7.4 at a scan rate of $60 \mathrm{deg} \mathrm{h}^{-1}$. The data points are shown as open circles $(O)$. The two-state tetramer self-dissociating model fit to the data is shown as a thin line. The calculated baseline is shown as a dotted line.

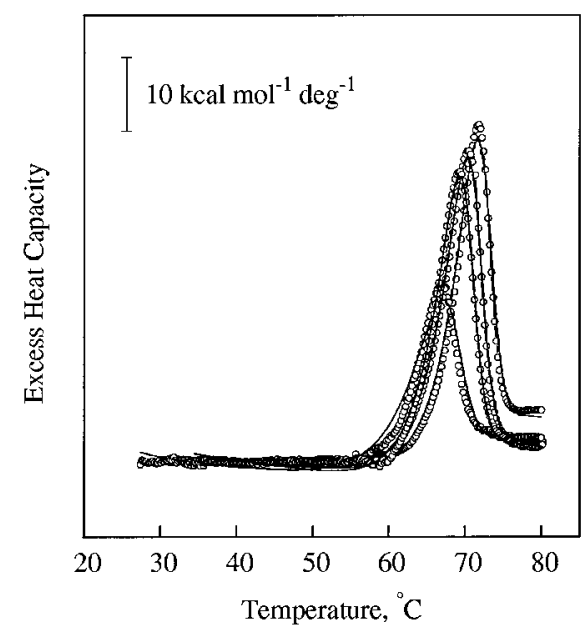

FIGURE 2: Excess heat capacity of $\mathrm{SecB}$ at different protein concentrations. SecB concentrations used from left to right are 16.9, 25.6, 53.2, and $86.4 \mu \mathrm{M}$. The data points are shown as open circles (O). The continuous lines represent theoretical curves generated with two-state unfolding coupled to subunit dissociation. The thermodynamic parameters corresponding to the fits are shown in Table 1.

Table 1: Thermodynamic Parameters for the Unfolding of the SecB Tetramer as a Function of Protein Concentration at $\mathrm{pH} 7.4$

\begin{tabular}{cccc}
\hline$[\mathrm{SecB}](\mu \mathrm{M})$ & $T_{\mathrm{m}}(\mathrm{K})$ & $\Delta H_{\mathrm{m}}{ }^{\alpha}\left(\mathrm{kcal} \mathrm{mol}^{-1}\right)$ & $\Delta C_{\mathrm{p}}{ }^{\alpha}\left(\mathrm{kcal} \mathrm{mol}^{-1} \mathrm{~K}^{-1}\right)$ \\
\hline 8.3 & 339.5 & 199 & $10.9( \pm 0.4)$ \\
16.9 & 339.8 & 208 & $9.4( \pm 0.4)$ \\
25.6 & 341.6 & 237 & $9.8( \pm 0.2)$ \\
53.2 & 342.8 & 283 & $11.7( \pm 0.3)$ \\
86.4 & 344.1 & 292 & $12.9( \pm 0.3)$ \\
\hline
\end{tabular}

${ }^{a}$ The average uncertainties in the measurements of the $\Delta H_{\mathrm{m}}$ and $\Delta C_{\mathrm{p}}$ are 1.1 and $3 \%$, respectively.

the data. This is in accordance with the tetrameric status of the SecB oligomer (21). There is cooperative unfolding of the tetramer to unfolded monomers without any detectable intermediates. Thermal transition curves at protein concentrations lower than $8.3 \mu \mathrm{M}$ were examined by $\mathrm{CD}$ and absorbance experiments, as the calorimetric response does not allow accurate measurements at such low concentrations. The change in ellipticity at $222 \mathrm{~nm}$ and increase in absor-

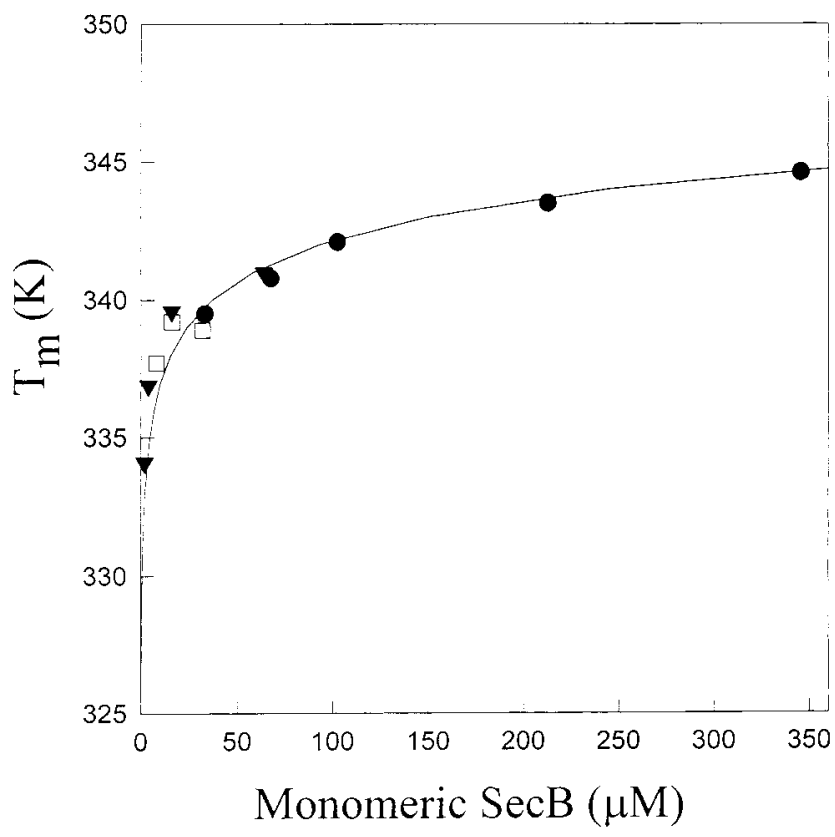

FIGURE 3: Unfolding transition temperature $\left(T_{\mathrm{m}}\right)$ of $\mathrm{SecB}$ as a function of tetramer concentration. The solid circles $(-)$ are from DSC-determined data, solid triangles $(\mathbf{\Lambda})$ are from $\mathrm{CD}$ experiments and the open squares $(\square)$ are from absorbance experiments. For all the data, the $T_{\mathrm{m}}$ is defined as the temperature at which $F_{\mathrm{u}}=$ 0.5 . The solid line is the predicted dependency according to the two-state tetramer self-dissociating model.

Table 2: Thermodynamic Parameters for the Unfolding of the SecB Tetramer as a Function of $\mathrm{pH}$

\begin{tabular}{cccc}
\hline $\mathrm{pH}$ & $T_{\mathrm{m}}(\mathrm{K})$ & $\Delta H_{\mathrm{m}}{ }^{\alpha}\left(\mathrm{kcal} \mathrm{mol}^{-1}\right)$ & $\Delta C_{\mathrm{p}}{ }^{\alpha}\left(\mathrm{kcal} \mathrm{mol}^{-1} \mathrm{~K}^{-1}\right)$ \\
\hline 9.50 & 327.1 & 100 & $8.5( \pm 0.6)$ \\
9.00 & 330.8 & 124 & $7.2( \pm 0.8)$ \\
8.75 & 332.9 & 141 & $10.7( \pm 0.7)$ \\
8.50 & 333.6 & 157 & $9.1( \pm 0.9)$ \\
8.38 & 334.5 & 177 & $5.8( \pm 0.4)$ \\
8.25 & 335.1 & 180 & $7.2( \pm 0.5)$ \\
8.00 & 338.6 & 198 & $8.7( \pm 0.3)$ \\
7.80 & 339.4 & 210 & $14.4( \pm 0.4)$ \\
7.40 & 340.9 & 238 & $13.7( \pm 0.2)$ \\
7.00 & 341.0 & 245 & $11.3( \pm 0.3)$ \\
6.50 & 341.3 & 256 & $12.7( \pm 0.2)$ \\
\hline
\end{tabular}

${ }^{a}$ The average uncertainties in the measurements of $\Delta H_{\mathrm{m}}$ and $\Delta C_{\mathrm{p}}$ are 1.6 and $5.5 \%$, respectively.

bance at $253 \mathrm{~nm}$ upon SecB unfolding were used to monitor unfolding of SecB. The unfolding monitored by change in absorbance and in secondary $\mathrm{CD}$ at the same concentration of protein is essentially overlapping, showing that the unfolding is truly a two-state process (data not shown). Another indicator of two-state unfolding is the fact that all the DSC transitions fit well to a single transition involving dissociation of the tetramer to unfolded monomers. We have measured the $\Delta H_{\mathrm{m}}$ as a function of $T_{\mathrm{m}}$ by carrying out DSC scans at different pHs. The DSC scans were carried out as a function of $\mathrm{pH}$ in the $\mathrm{pH}$ range 6.5-9.5 and the results are summarized in Table 2. Figure 4 shows the baselinesubtracted fits at two extremes of $\mathrm{pH} 6.75$ and 9. Below $\mathrm{pH}$ 8.75 , all DSC scans could be well fit to subunit dissociation coupled to unfolding where $n=4$. Above $\mathrm{pH} 8.75$, the DSC scans were found to fit both $n=2$ and $n=4$ equally well (Figure 4). This is in accordance with a previous observation that dimers are populated at alkaline $\mathrm{pH}(21)$. In all the cases, the data are well fit to a two-state model. The free energy 


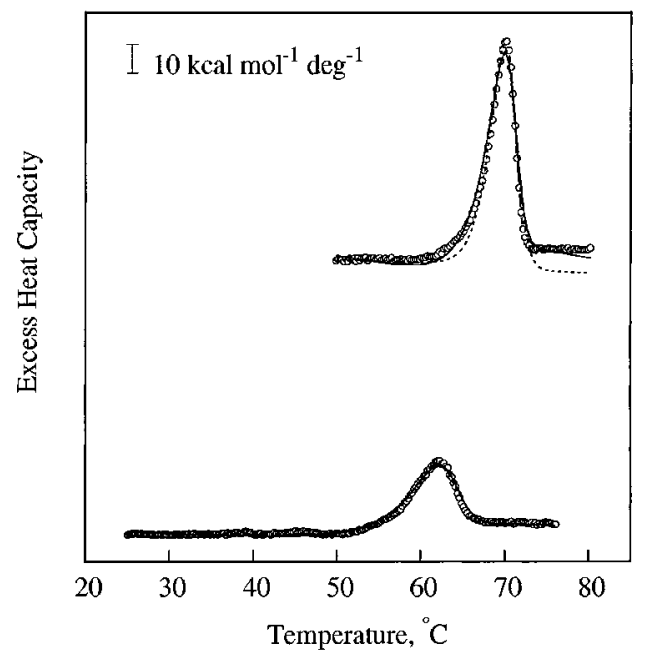

Figure 4: DSC scans of $25 \mu \mathrm{M} \mathrm{SecB}$ at pH 6.5 (bottom) and 9 (top). The data points are shown as open circles (O). The twostate self-dissociating model fit to the data is shown as a thin solid line for $n=4$ and dotted line for $n=2$.

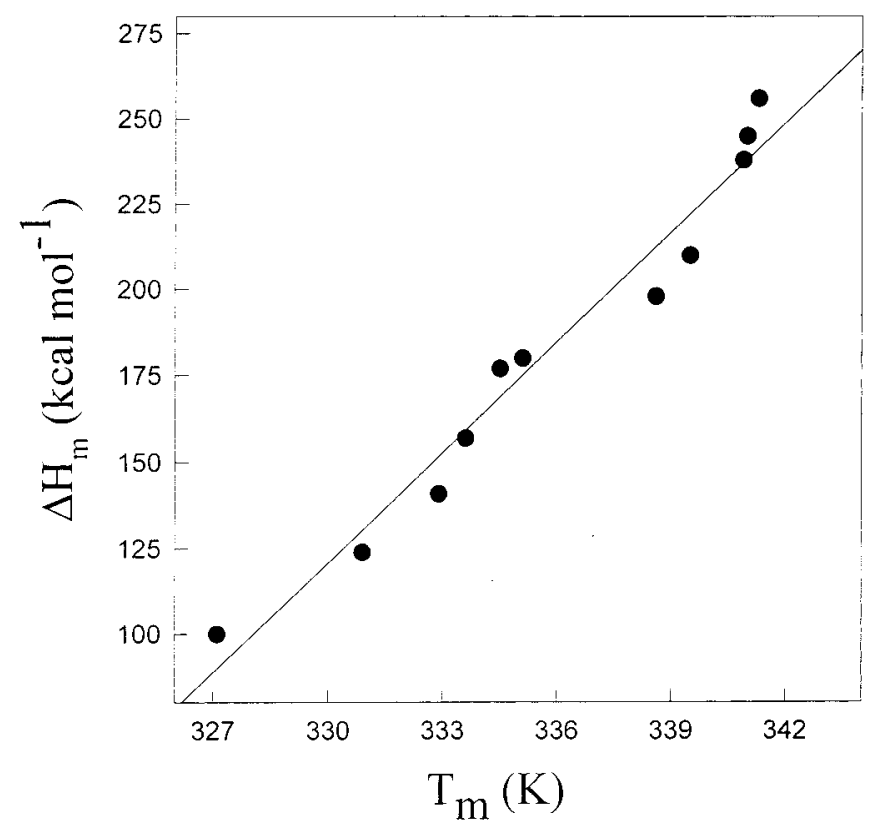

FIGURE 5: $\Delta C_{\mathrm{p}}$ estimation from the linear dependence of $\Delta H_{\mathrm{m}}$ on $T_{\mathrm{m}}$. The $\Delta H_{\mathrm{m}}$ and $T_{\mathrm{m}}$ values from Table 2 are fitted to a straight line. The slope yields a $\Delta C_{\mathrm{p}}$ value of $10.7 \pm 0.7 \mathrm{kcal} \mathrm{mol}^{-1} \mathrm{~K}^{-1}$.

of unfolding of a protein can be obtained as a function of temperature from a single DSC experiment. However, it is difficult to get an accurate estimate of the $\Delta C_{\mathrm{p}}$ from a single scan (22). We have estimated the $\Delta C_{\mathrm{p}}$ value from a plot of $\Delta H_{\mathrm{m}}$ as a function of $T_{\mathrm{m}}$ (Figure 5) The slope $\left(\mathrm{d} \Delta H_{\mathrm{m}} / \mathrm{d} T_{\mathrm{m}}\right)$ provides an estimate of $\Delta C_{\mathrm{p}}$ of $10.7 \pm 0.7 \mathrm{kcal} \mathrm{mol}^{-1} \mathrm{~K}^{-1}$. The data fit well to a straight line (correlation coefficient 0.96). This indicates that $\Delta C_{\mathrm{p}}$ is independent of temperature and $\mathrm{pH}$ within the range of this study.

Model for Two-State Unfolding of SecB. A two-state concentration-dependent self-dissociating model for thermal unfolding shown below was used to analyze the data as described $(23,14)$. In the case of folded SecB $\left(\mathrm{S}_{4}\right)$, unfolding to four unfolded monomers (U) with no intermediate states the transition is described by

$$
\mathrm{S}_{4} \Leftrightarrow 4 \mathrm{U}
$$

$$
\begin{gathered}
K=\left[\mathrm{U}^{4} /\left[\mathrm{S}_{4}\right]\right. \\
\Delta G_{\mathrm{u}}^{\circ}(T)=-R T \ln K \\
\Delta G_{\mathrm{u}}^{\circ}(T)=\Delta H^{\circ}\left(T_{\mathrm{o}}\right)-T \Delta S\left(T_{\mathrm{o}}\right)+\Delta C_{\mathrm{p}}\left(T-T_{\mathrm{o}}-\right. \\
T \ln \left(T / T_{\mathrm{o}}\right)
\end{gathered}
$$

where $K$ is defined as the equilibrium constant for the tetrameric state converting directly to the (monomeric) unfolded state, without folded monomeric intermediate, and $T_{\mathrm{o}}$ is some reference temperature. The total protein concentration $\left(P_{\mathrm{T}}\right)$ in monomer units can be expressed as

$$
P_{\mathrm{T}}=[\mathrm{U}]+\left[\mathrm{S}_{4}\right] 4
$$

The fractional populations in the native $\left(F_{\mathrm{N}}\right)$ and unfolded $\left(F_{\mathrm{U}}\right)$ states are given by

$$
F_{\mathrm{N}}=4\left[\mathrm{~S}_{4}\right] / P_{\mathrm{T}}
$$

Using eqs 3-6 we obtain,

$$
\begin{gathered}
F_{\mathrm{U}}=[\mathrm{U}] / P_{\mathrm{T}} \\
F_{\mathrm{U}}^{4}+K F_{\mathrm{U}} / 4 P_{\mathrm{T}}^{4}-K / 4 P_{\mathrm{T}}^{4}=0
\end{gathered}
$$

Such a model would predict a dependence of $T_{\mathrm{m}}$ (the temperature at which $F_{\mathrm{U}}$ is 0.5 ) on the monomeric SecB concentration. Figure 3 shows the $T_{\mathrm{m}}$ as a function of monomeric SecB concentration. The solid line in Figure 3, which predicts the dependence of the $T_{\mathrm{m}}$ as a function of SecB concentration, was generated in the following way. At each temperature $T$ in the range $250-400 \mathrm{~K}$, values of $\Delta G^{\circ}{ }_{\mathrm{u}}$ and $K$ are calculated from eqs 4 and 3, respectively, using the value of $\Delta C_{\mathrm{p}}$ of $10.7 \mathrm{kcal} \mathrm{mol}^{-1}, \Delta H^{\circ}\left(T_{\mathrm{o}}\right)$ and $\Delta S\left(T_{\mathrm{o}}\right)$ determined from calorimetric data using $341 \mathrm{~K}$ as reference temperature. The value of $K$ is substituted into eq 8 with $F_{\mathrm{U}}$ $=0.5$ to give a corresponding value for $P_{\mathrm{T}}$. As seen from Figure 3 the experimental data fits well to $T_{\mathrm{m}} \mathrm{s}$ predicted from the self-dissociation coupled with unfolding model. These studies show that $\mathrm{SecB}$ appears to unfold as a single cooperative unit and that unfolding of the monomers is coupled to subunit dissociation. Such reversible two-state unfolding thermal unfolding behavior has been shown in only one other multimeric chaperone system: co-chaperonin GroES. GroES is a heptamer of $10 \mathrm{kDa}$ subunits and the thermal unfolding of GroES has been shown to involve a highly cooperative reversible transition between folded heptamers and unfolded monomers (15). The thermodynamics of unfolding studies of chaperone GroES has provided insights into the stabilization and assembly of these large multisubunit structures. Analysis of the high-resolution structure of GroES indicated that the isolated folded monomers buried a significantly smaller fraction of their total surface than monomeric proteins of similar molecular mass. Thus the stabilization of the oligomeric structure was derived from intersubunit interactions rather than intramolecular interactions (15). In the cases of dimeric proteins, Arc repressor and core histone $\mathrm{H} 2 \mathrm{~A}-\mathrm{H} 2 \mathrm{~B}$, intersubunit interactions have been shown to stabilize the dimeric structure (13, 24). 


\begin{tabular}{|c|c|c|c|c|c|c|c|}
\hline protein & $N_{\text {res }}{ }^{a}$ & $\begin{array}{l}\text { oligomeric } \\
\text { state }\end{array}$ & $\begin{array}{c}\Delta C_{\mathrm{p}} \\
{\left[\mathrm{kcal}(\mathrm{mol} \mathrm{K})^{-1}\right]}\end{array}$ & $\begin{array}{c}\Delta C_{\mathrm{p}} \\
{\left[\mathrm{cal}(\mathrm{mol} \mathrm{K} \text { residues })^{-1}\right]}\end{array}$ & $\begin{array}{l}\Delta G^{\circ}{ }_{\mathrm{u}}(298 \mathrm{~K}) \\
\left(\mathrm{kcal} \mathrm{mol}^{-1}\right)\end{array}$ & $\begin{array}{c}\Delta G^{\circ}{ }_{\mathrm{u}}(298 \mathrm{~K}) \\
{\left[\mathrm{cal}(\text { mol residues })^{-1}\right]}\end{array}$ & ref \\
\hline BZIP GCN4 & 112 & dimer & 0.7 & 6.5 & 13.5 & 120.8 & 31 \\
\hline ROP & 126 & dimer & 2.5 & 19.5 & 17.1 & 135.7 & 29 \\
\hline Arc repressor & 106 & dimer & 1.6 & 15.1 & 11 & 103.8 & 13 \\
\hline adenylate kinase & 582 & trimer & 2.9 & 4.9 & 30 & 51.5 & 27 \\
\hline HIV protease & 198 & dimer & 3.2 & 16.2 & 14.2 & 71.7 & 16 \\
\hline $\mathrm{H} 2 \mathrm{~A}-\mathrm{H} 2 \mathrm{~B}$ & 255 & dimer & 2.8 & 11 & 8.4 & 32.9 & 24 \\
\hline p53 & 252 & tetramer & 1.7 & 6.7 & 30.3 & 120.2 & 14 \\
\hline Melltin & 104 & tetramer & 1.2 & 11.5 & $\mathrm{NR}^{b}$ & NR & 23 \\
\hline archaeal histones & 138 & dimer & 2 & 14.4 & 14 & 101.4 & 32 \\
\hline GroES & 679 & heptamer & 7 & 10.3 & 63 & 92.8 & 15 \\
\hline SecB & 620 & tetramer & 10.6 & 17 & 27.9 & 45.2 & \\
\hline
\end{tabular}

${ }^{a} N_{\text {res }}$ is the total number of residues in the oligomer. ${ }^{b} \mathrm{NR}$, not reported. All thermodynamic parameters are expressed per mole oligomer.

Comparison of $\Delta C_{p}$ of $\operatorname{Sec} B$ with Other Multimeric Proteins. Thermal unfolding studies have been extensively carried out on small monomeric proteins because their unfolding transitions are highly reversible. However, there are fewer studies on the thermal unfolding of multimeric proteins because of their tendency to undergo irreversible denaturation. SecB is a large tetrameric protein, which displays two-state reversible, thermal folding. It is therefore of interest to see how the thermodynamic parameters governing SecB unfolding compare with other large monomeric and multimeric proteins. $\Delta C_{\mathrm{p}}$ is the thermodynamic quantity closely related to the hydrophobic driving force and the burial of nonpolar surface area upon folding. Using the data on unfolding thermodynamics of small monomeric proteins, attempts have been made to correlate the total accessible surface area $(\triangle \mathrm{ASA})$ or the nonpolar surface $\left(\triangle \mathrm{ASA}_{\mathrm{np}}\right)$ buried upon unfolding and $\Delta C_{\mathrm{p}}(25)$. The extent of polar and nonpolar surfaces that are buried from the solvent is a major determinant of the stability of the native state. In the case of the p53 tetramer, the tetramerization domain buries a larger fraction of nonpolar surface than most globular proteins (14). Similar observations have been made in case of the assembly of heptameric GroES and coiled coil GCN4 $(15,26)$. According to Myers et al. (25) for monomeric proteins, $\triangle \mathrm{ASA}$ is linearly dependent on the number of residues $\left(N_{\text {res }}\right)$ in the protein and $\Delta C_{\mathrm{p}}$ is linearly dependent on the $\triangle \mathrm{ASA}$ as follows:

$$
\begin{gathered}
\Delta \mathrm{ASA}=-907+93 N_{\text {res }} \\
\Delta C_{\mathrm{p}}=-119+0.2 \Delta \mathrm{ASA}
\end{gathered}
$$

Hence,

$$
\Delta C_{\mathrm{p}}=-300.4+18.6 N_{\text {res }} \mathrm{cal} \mathrm{mol}^{-1} \mathrm{~K}^{-1}
$$

The empirical eq 11 given by Myers et al. (25) is based on a correlation analysis of the data from 45 monomeric proteins. It would, thus, be of interest to examine if oligomeric proteins behave in a similar fashion. In a recent study, Backmann et al. (27) reported an analysis of the calculated versus experimentally observed $\Delta C_{\mathrm{p}}$ values. They concluded that $\Delta C_{\mathrm{p}}$ values of oligomeric proteins reported in the literature tend to be low values compared with the values calculated by eq 11 . Karantza et al. (28) have suggested that incomplete unfolding is responsible for the relatively low heat capacity change. High values of $\Delta C_{\mathrm{p}}$ were found for only a few oligomeric proteins $(13,29,30)$. In the case of the SecB tetramer for which the $N_{\text {res }}$ is 620 , we obtain a $\Delta C_{\mathrm{p}}$ value of $11.2 \mathrm{kcal} \mathrm{mol}^{-1} \mathrm{~K}^{-1}$ from eq 11 . This is close to the experimentally determined value of $10.7 \pm 0.7$ $\mathrm{kcal} \mathrm{mol}{ }^{-1} \mathrm{~K}^{-1}$. This suggests that the unfolded state of SecB is probably not compact and is devoid of any structure contributing to the heat capacity change. Table 3 summarizes the thermodynamic data for a few oligomeric proteins that undergo unfolding coupled with subunit dissocation. As seen from Table 3, the $\Delta C_{\mathrm{p}}$ per moles of residue for SecB is 17.2 cal $\operatorname{deg}^{-1}(\text { mol residue })^{-1}$. This is higher than for typical small monomeric proteins and also the listed multimeric proteins. This suggests that a large protein such as the SecB tetramer, buries a larger fraction of its total ASA than smaller proteins. It has been suggested previously that $\mathrm{SecB}$ is a malleable macromolecule, which may be converted to a rigid state upon interaction with target substrates (33). The present data suggests instead that SecB has a well packed tetrameric structure, even in the absence of a bound substrate.

Oligomeric Status of SecB in the Cell. The concentration of SecB in vivo has been estimated to be $4 \mu \mathrm{M}(34,35)$. Gel filtration experiments even at the lowest possible concentrations (nanomolar) were unable to detect the presence of any intermediate dimeric species. Hence, it was concluded that that the equilibrium constant for the conversion to dimeric species is lower than nanonmolar (35). The stability curve, which represents the variation with temperature of the change in the standard Gibbs energy of unfolding, is shown in Figure 6. The $\Delta G^{\circ}{ }_{\mathrm{u}}(298)$ per SecB tetramer at $298 \mathrm{~K}$ as estimated from the stability curve is found to be $27.9 \pm 2 \mathrm{kcal} \mathrm{mol}^{-1}$. Thus, at $298 \mathrm{~K}$, the equilibrium constant between dissociated, unfolded monomers and folded tetramers is $10^{-20} \mathrm{M}^{3}$. The present data suggests that, under physiological conditions, the functional form of $\mathrm{SecB}$ is stable as a tetramer within the cell. The temperature of maximal stability of SecB is $314 \mathrm{~K}$. This is higher than for smaller, monomeric globular protein predictions, consistent with a recent prediction of the size dependence of this parameter (36). All the thermodynamic data suggest that the $\mathrm{SecB}$ tetramer is a tightly packed, well-folded and stable protein. Hence, substrate binding is likely to occur on the surface rather than in an interior cavity as in the case of the GroEL/GroES system.

Denaturant-Mediated Unfolding and Reactivity of Cys Residues in $\mathrm{SecB}$. Equilibrium unfolding of chaperone SecB was carried out using urea and $\mathrm{GdnHCl}$ in $\mathrm{CGH} 10, \mathrm{pH}$ 7.4. Intrinsic protein fluorescence methods and reactivity of the cysteine residues to the thiol specific reagent DTNB was 


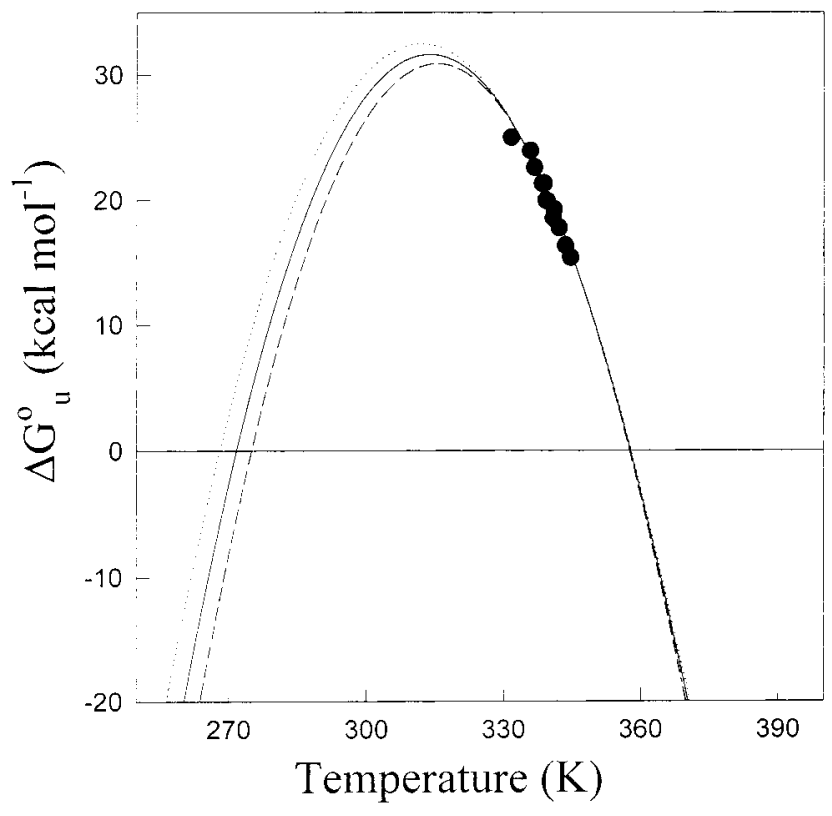

FiguRE 6: $\Delta G^{\circ}$ for unfolding of $\operatorname{SecB}$ as a function of temperature at $\mathrm{pH}$ 7.4. The stability curve (solid line) was calculated from eq 4 using the following parameters: $\Delta H^{\circ}\left(T_{\mathrm{o}}\right)=323.6 \mathrm{kcal} \mathrm{mol}^{-1}$, $\Delta S\left(T_{\mathrm{o}}\right)=0.890 \mathrm{kcal} \mathrm{mol}^{-1} \mathrm{~K}^{-1}, T_{\mathrm{o}}=341 \mathrm{~K}$, and $\Delta C_{\mathrm{p}}=10.7$ $\mathrm{kcal} \mathrm{mol}^{-1} \mathrm{~K}^{-1}$. The two dotted lines represent the effect on the stability curve due the error in the measurement of $\Delta C_{\mathrm{p}}$ of \pm 0.7 $\mathrm{kcal} \mathrm{mol}{ }^{-1} \mathrm{~K}^{-1}$. The filled circles $(\bullet)$ represent values of $\Delta G^{\circ}{ }_{\mathrm{u}}$ for unfolding using eqs 8 and 3 obtained using experimentally observed $T_{\mathrm{m}} \mathrm{s}$ at various $P_{\mathrm{T}} \mathrm{s}$.

used to monitor the denaturant mediated unfolding of SecB. Figure 7A shows the unfolding of $\mathrm{SecB}$ as monitored by the change in fluorescence at $358 \mathrm{~nm}$. As evident from the figure, there is an increase in fluorescence on unfolding of SecB. Folded SecB exhibits an emission maximum at 305 $\mathrm{nm}$ while unfolded $\mathrm{SecB}$ has an emission maximum at 355 $\mathrm{nm}$ when excited at $280 \mathrm{~nm}$ (data not shown). However, in both urea and $\mathrm{GdnHCl}$-mediated unfolding, the transition was found to be irreversible as monitored by fluorescence methods. The formation of soluble aggregates could lead to irreversible unfolding of SecB. We tested this hypothesis by carrying out gel filtration experiments as a function of denaturant concentration. In the absence of any denaturants, tetrameric SecB elutes at $13.7 \mathrm{~mL}$ in a Superose 6 column. Bruce et al. (21) have detected dimers of SecB at alkaline $\mathrm{pH}$. We have also been able to fit DSC scans to $n=2$ for experiments in the alkaline range. At concentrations lower than $0.75 \mathrm{M}$ of $\mathrm{GdnHCl} \mathrm{SecB}$ elutes also at $13.7 \mathrm{~mL}$. Muren et al. (35) have reported mutations in SecB which were able to populate dimers of $\mathrm{SecB}$ and result in loss of $\mathrm{SecB}$ function. We have not been able to detect dimers of SecB at low concentration of denaturants by gel filtration experiments (data not shown). At concentrations higher than $1.2 \mathrm{M}$ $\mathrm{GdnHCl}$, SecB aggregates and elutes at $8 \mathrm{~mL}$, which is within the void volume of the column (data not shown). Another method used to monitor the denaturant-mediated unfolding reaction was the reactivity of cysteine residues to thiol specific agents. We have used DTNB and DTP as thiol solvent accessibility probes in our experiments. The reactivity of cysteine has been used previously to monitor the kinetics of protein unfolding. Also, equilibrium unfolding and refolding of proteins has been studied by monitoring the reactivity of cysteine residues (37-39). DTNB reacts with solvent

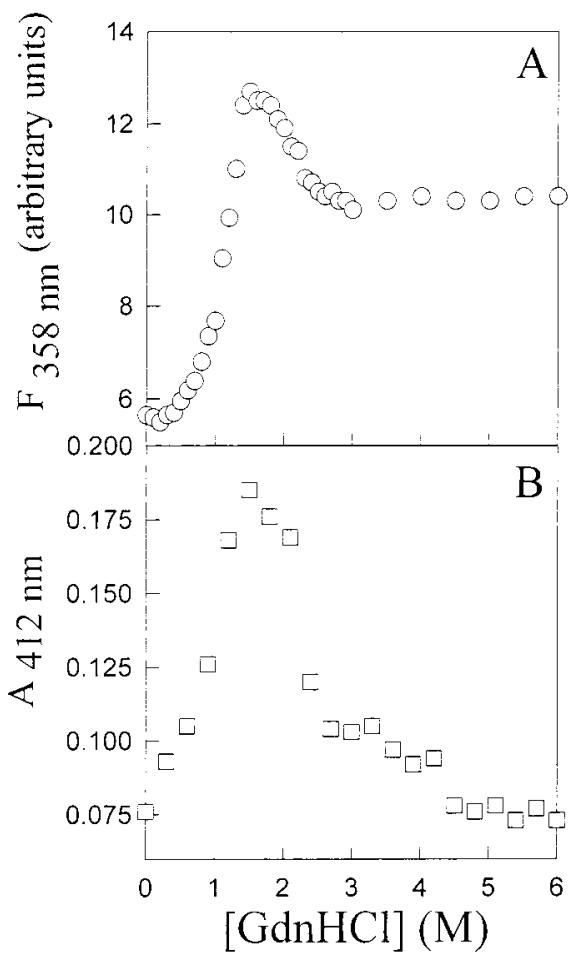

FIGURE 7: (A) GdnHCl denaturation curve of $1.1 \mu \mathrm{M}$ SecB at 25 ${ }^{\circ} \mathrm{C}$ and $\mathrm{pH}$ 7.4. GdnHCl-induced denaturation was followed by monitoring the fluorescence emission at $358 \mathrm{~nm}$ on excitation at $280 \mathrm{~nm}$. (B) DTNB reaction of the thiols of $1.1 \mu \mathrm{M} \mathrm{SecB}$ incubated overnight in $\mathrm{GdnHCl}$ at $25^{\circ} \mathrm{C}$ and $\mathrm{pH}$ 7.4. The reaction with DTNB was carried out for $4 \mathrm{~h}$ and then the absorbance at $412 \mathrm{~nm}$ was recorded. An absorbance of 0.06 at $412 \mathrm{~nm}$ corresponds to 4 thiols labeled per tetramer of $\mathrm{SecB}$ under these conditions.

accessible free cysteine in proteins by a thiol-disulfide exchange reaction. The increase in absorbance at $412 \mathrm{~nm}$ due to the TNB ion released can be used to estimate the number of thiols labeled in the protein (19). After overnight incubation at a given denaturant concentration, samples of SecB were assayed for thiol reactivity by incubating the protein with DTNB for $4 \mathrm{~h}$. As shown in Figure 7B the number of Cys labeled increases with increase in denaturant concentration up to $1.5 \mathrm{M} \mathrm{GdnHCl}$ and $2 \mathrm{M}$ Urea. However beyond $1.5 \mathrm{M} \mathrm{GdnHCl}$ and $2 \mathrm{M}$ urea, there is a decrease in the number of Cys getting labeled, probably because of the formation of aggregates. Cys in different environments should react with different rates and hence would exhibit multiexponential kinetics of reactivity to DTNB (40). SecB has four Cys per monomer. The reactivity of the four Cys residues per monomer was assayed by reaction with DTNB as a function of denaturant and salt concentration. The kinetics of unfolding as monitored by reactivity of the thiols to DTNB was carried out. Below $1 \mathrm{M}$ of $\mathrm{GdnHCl}, 1$ thiol per monomer reacts with DTNB (data not shown). As shown in Figure 8, upon an increase in the $\mathrm{GdnHCl}$ concentration beyond $1 \mathrm{M}$, all 16 thiols in SecB tetramer are labeled. The labeling occurs with slow multiexponential kinetics showing that the Cys are still in different environments and the protein is unfolding while labeling is occurring. At high denaturant concentration, the reaction becomes single exponential, showing that the environment of all the Cys residues is the same, i.e., that complete unfolding has occurred before labeling. The reactivity of the four Cys, which are solvent exposed, was found to be dependent on salt concentration. 


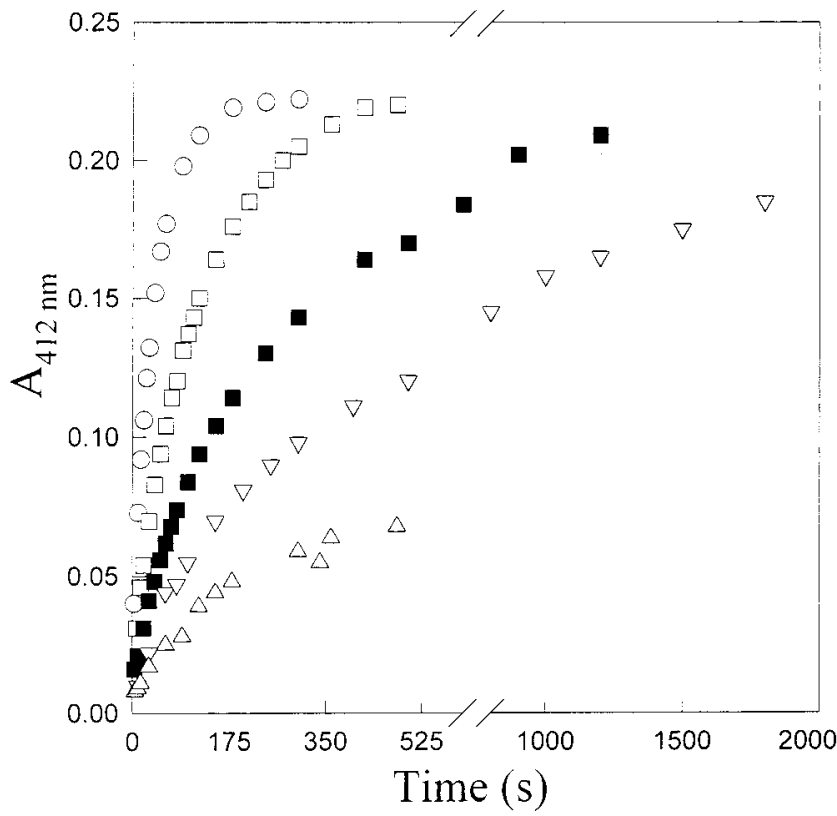

FIGURE 8: Unfolding kinetics as a function of $\mathrm{GdnHCl}$ as monitored by the reactivity of exposed thiols. Kinetic traces of unfolding of $\mathrm{SecB}$ as monitored by increase in absorbance at $412 \mathrm{~nm}$ at $1 \mathrm{M}$ $(\triangle), 2 \mathrm{M}(\nabla), 3 \mathrm{M}(\mathbf{\square}), 4 \mathrm{M}(\square)$, and $5.2 \mathrm{M}(\bigcirc) \mathrm{GdnHCl}$ concentrations contained in CGH10 pH 7.4. A $100 \mu \mathrm{L}$ solution of $6.6 \mu \mathrm{M} \mathrm{SecB}$ was added to a $500 \mu \mathrm{L}$ solution of $200 \mu \mathrm{M}$ DTNB contained in appropriate concentration of $\mathrm{GdnHCl}$ in $\mathrm{CGH} 10, \mathrm{pH}$ 7.4 , such that the final denaturant concentration would be as stated above. An absorbance of 0.06 at $412 \mathrm{~nm}$ corresponds to four thiols labeled per tetramer of SecB under these conditions.

As shown in Figure 9A, in low ionic strength buffer CGH10, $\mathrm{pH}$ 7.4, the four Cys react slowly with multiexponential kinetics. With increase in the $\mathrm{KCl}$ concentration the reaction can be well fit to a single exponential function. Thus, at low ionic strength the environment surrounding the four Cys is not apparently identical. However, in the presence of 100 $\mathrm{mM} \mathrm{KCl}$ in CGH10, $\mathrm{pH} 7.4$, the reaction fits well to a single exponential function, indicating the environment surrounding the Cys has become identical. Such salt-dependent reactivity can be explained as follows: DTNB is a negatively charged molecule. If one assumes that the four Cys are in close proximity, the reaction of the first Cys would be fast and the reaction of the subsequent Cys would be slower due to the presence of the negatively charged carboxylate of TNB on the reacted Cys (40). As the negative charge is screened due to the presence of salt, the reaction becomes single exponential. Hence, the apparent rate constant increases from $0.0013(100 \mathrm{mM} \mathrm{KCl})$ to $0.0042(800 \mathrm{mM} \mathrm{KCl})$. As shown in Figure 9B, the reactivity of the uncharged molecule, DTP, is independent of salt concentration and fits well to a single exponential (apparent rate constant is 0.013). This suggests that the environments surrounding the solvent-exposed Cys in the SecB tetramer are very similar or identical. Also, the reactivity of the both thiol-reactive probes indicates that the cysteines are partly accessible to solvent. This observation coupled with observations that thermal unfolding is two state and that $\mathrm{SecB}$ contains four identical binding sites for small unfolded substrates (34) suggests that, at neutral $\mathrm{pH}, \mathrm{SecB}$ is a symmetrical tetramer. This differs from results from mutational studies (35), which suggested that SecB is a dimer of dimers. In the present experiments, we do not observe dimeric intermediates in SecB unfolding. It is only at alkaline
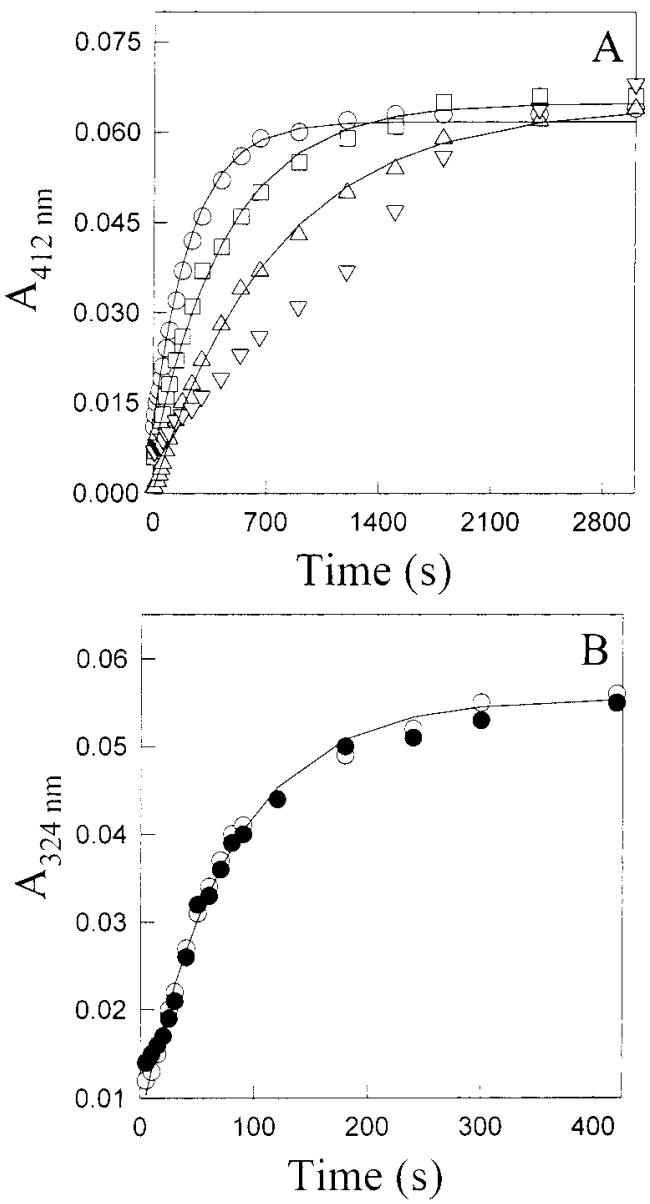

FIGURE 9: (A) Salt dependence of the reactivity of the solvent exposed Cys to DTNB. The reaction kinetics of DTNB to the exposed Cys in native $\operatorname{SecB}(1.1 \mu \mathrm{M})$ was monitored at $412 \mathrm{~nm}$ as a function of $\mathrm{KCl}$ in $\mathrm{CGH} 10, \mathrm{pH}$ 7.4. (O) $600 \mathrm{mM} \mathrm{KCl,} \mathrm{( \square )} 200$ $\mathrm{mM} \mathrm{KCl},(\triangle) 100 \mathrm{mM} \mathrm{KCl}$, and $(\nabla) 0 \mathrm{mM} \mathrm{KCl}$. An absorbance of 0.06 at $412 \mathrm{~nm}$ corresponds to four thiols labeled per tetramer of SecB under conditions stated. (B) Reaction kinetics of the solvent exposed Cys in $\mathrm{SecB}(0.7 \mu \mathrm{M})$ to DTP was carried out in $0 \mathrm{mM}$ $\mathrm{KCl}(\bullet)$ and $600 \mathrm{mM} \mathrm{KCl} \mathrm{(O)} \mathrm{contained} \mathrm{in} \mathrm{CGH10,} \mathrm{pH} 7.4$, in an identical manner as stated in panel A. However, the reaction kinetics was monitored at $324 \mathrm{~nm}$. An absorbance of 0.055 at $324 \mathrm{~nm}$ corresponds to 4 thiols labeled per tetramer of $\mathrm{SecB}$ under conditions stated. The solid lines in both panels A and B represent single-exponential fits to individual traces.

$\mathrm{pH}$ that the denaturation data fit equally well to both tetramer and dimer dissociation models. The thiol reactivity data are qualitative in nature, and hence it is still possible that $\mathrm{SecB}$ may be a dimer of dimers, and rather than a symmetrical tetramer, a definitive answer must await structure determination of SecB by crystallography or NMR.

\section{ACKNOWLEDGMENT}

We thank Prof. B. de Kruijff for kindly providing the SecB expression plasmid pJW25. We thank C. Ganesh and Suvobrata Chakravarty for helpful discussions. Microcal MC-2 is provided by the Department of Science \& Technology, Government of India. C.P.S. is a Research Associate of the Council of Scientific and Industrial Research, Government of India.

\section{REFERENCES}

1. Gething, M., and Sambrook, J. (1992) Nature 355, 33-44.

2. Hartl, F. U. (1996) Nature 381, 571-580. 
3. Weiss, J. B., and Bassford, P. J. (1990) J. Bacteriol. 172, 3023-3029.

4. Randall, L., and Hardy, S. J. (1986) Cell 46, 921-928.

5. Scheibel, E., Driessen, A. J., Hartl, F. U., Wickner, W. (1991) Cell 64, 927-939.

6. Lecker, S., Lill, R., Ziegelhoffer, T., Georgopoulos, C., Bassford, P. J., Kumamoto, C. A., and Wickner, W. (1989) EMBO J. 8, 2703-2709.

7. Hardy, S. J., and Randall, L. L. (1991) Science 251, 439443.

8. Lecker, S. H., Driessen, J. M., and Wickner, W. (1990) EMBO J. 9, 2309-2314.

9. Panse, V. G., Udgaonkar, J. B., and Varadarajan, R. (1998) Biochemsitry 37, 14477-14483.

10. Becktel, W. J., and Schellman, J. A. (1987) Biopolymers 26, 1859-1877.

11. Privalov, P. L. (1979) Adv. Protein Chem. 33, 167-241.

12. Murphy, K. P., and Freire, E. (1992) Adv. Protein Chem. 43, $313-261$.

13. Bowie, J. U., and Sauer, R. T. (1989) Biochemistry 28, 71397143.

14. Johnson, C. R., Morin, P. E., Arrowsmith, C. H., and Freire, E. (1995) Biochemistry 34, 5309-5316.

15. Boudker, O., Todd, M. J., and Freire, E. (1997) J. Mol. Biol. 272, 770-779.

16. Todd, M. J., Semo, N., and Freire, E. (1998) J. Mol. Biol. 283, 475-488.

17. Laemmli, U. (1970) Nature 227, 680-685.

18. Fasman, G. D., Park, K., and Randall, L. L. (1995) J. Protein Chem. 14, 595-600.

19. Ramachandran, S., and Udgaonkar J. B. (1996) Biochemistry $35,8876-8785$.

20. Pace, C. N., and Laurents, D. V. (1989) Biochemistry 28, 2520-2525.

21. Bruce, J. E., Smith, V. F., Liu, C., Randall, L. L., and Smith, R. D. (1998) Protein Sci. 7, 1180-1185.

22. Ladbury, J. E., Kishore, N., Hellinga, H. W. Wynn, R., and Sturtevant, J. (1994) Biochemistry 33, 3688-3692.

23. Hagihara, Y., Oobatake, M., and Goto, Y. (1994) Protein Sci. 3, 1418-1429.
24. Karantza, V., Baxevonis, A. D., Freire, E., and Moudrianakis, E. N. (1995) Biochemistry 34, 5988-5996.

25. Myers, J. K., Pace, N. C., and Scholtz, J. M. (1995) Protein Sci. 4, 2138-2148.

26. Kenar, K. T., Garcia-Moreno, B., and Freire, E. (1995) Protein Sci. 4, 1934-1938.

27. Backmann, J., Schafer, G., Wyns, L., and Bonisch, H. (1998) J. Mol. Biol. 284, 817-833.

28. Karantza, V., Freire, E., and Moudrianakis, E. N. (1996) Biochemistry 35, 2037-2046.

29. Stief, C., Weber, P., Hinz, H. J., Flossdorf, J., Cesareni, G., and Kokkinides, M. (1993) Biochemistry 32, 3867-3876.

30. Jelesarov, I., and Bosshard, H. R. (1996) J. Mol. Biol. 263, $344-358$.

31. Thompson, K. S., Vinson, C. R., and Freire, E. (1993) Biochemistry 32, 5491-5496.

32. Li, W.T., Grayling, R. A., Sandman, K., Edmondson, S., Shriver, J. W., Reeve, J. N. (1998) Biochemistry 37, 1056310572.

33. Morimoto, R. I., Tisseres, A., and Georgopoulos, C. (1994) The Biology of Heat shock proteins and Molecular chaperones, pp 258-297, Cold Spring Harbor Laboratory press, Plainview, NY.

34. Fekkes, P., den Blaauwen, T., and Driessen, A. J. (1995) Biochemistry 34, 10078-10085.

35. Muren, E. M., Suciu, D., Topping, T. B., Kumamoto, C. A., and Randall, L. L. (1999) J. Biol. Chem. 274, 19397-19402.

36. Ganesh, C., Eswar, N., Srivastava, S., Ramakrishnan, C., and Varadarajan, R. (1999) FEBS Lett. 454, 31-36.

37. Goto, Y., and Hamaguchi, K. (1982) J. Mol. Biol. 156, $911-$ 926.

38. Ha, J. H., and Loh, S. N. (1998) Nat. Struct. Biol. 5, 730737.

39. Ballery, N., Desmadril, M., Minard, P., and Yon, J. M. (1993) Biochemistry 32, 708-714.

40. Bulaj, G., Kortemme, T., and Goldenberg, D. P. (1998) Biochemistry 37, 8965-8972.

BI992484L 